This document is the accepted manuscript version of the following article:

Shapovalova, S. O., Guda, A. A., Bubnov, M. P., Smolentsev, G., Rusalev, Y. V., Shapovalov, V. V., ... Soldatov, A. V. (2021). Temperature and time-resolved XANES studies of novel valence tautomeric cobalt complex. Chemistry Letters, 50(11), 1933-1937. https://doi.org/10.1246/c1.210426

1

Temperature and time-resolved XANES studies of novel valence tautomeric cobalt complex

\author{
S. O. Shapovalova ${ }^{1 *}$, A. A. Guda ${ }^{*}$, M. P. Bubnov ${ }^{2}$, G. Smolentsev ${ }^{3}$, Yu. V. Rusalev ${ }^{1}$, V. V. Shapovalov ${ }^{1}$, A. A. Zolotukhin ${ }^{2}$, V. K. \\ Cherkasov $^{2}$, A. G. Starikov ${ }^{4}$, V.G. Vlasenko ${ }^{5}$, A. V. Soldatov ${ }^{1}$ \\ ${ }^{1}$ The Smart Materials Research Institute, Sladkova 178/24, 344090 Rostov-on-Don, Russia \\ ${ }^{2}$ G. A. Razuvaev Institute of Organometallic Chemistry, Russian Academy of Sciences, 49 Tropinina Str., GSP-445, 603950 Nizhny \\ Novgorod, Russia \\ ${ }^{3}$ Paul Scherrer Institute, Villigen, 5232, Switzerland \\ ${ }^{4}$ Institute of Physical and Organic Chemistry, Southern Federal University, Stachki ave. 194/2, 344090, Rostov-on-Don, Russian \\ Federation \\ ${ }^{5}$ Institute of Physics, Southern Federal University, Stachki Ave., 194, 344090, Rostov-on-Don, Russia
}

E-mail: A.A.Guda guda@sfedu.ru,S.O. Shapovalova cherkasova.svetlana.o@gmail.com

$\begin{array}{ll}1 & \text { VT transition from the high-spin Co } \mathrm{Co}^{\mathrm{II}} \text { to low-spin Co } \mathrm{Co}^{\mathrm{III}} \\ 2 & \text { state was observed for complex (N-cyclo-hexyl-2- } \\ 3 & \text { iminopyridine)(3,6-di-tert-butyl-o-benzosemiquinonato)(3,6 } \\ 4 & \text {-di-tert-butyl-catecholato)cobalt in both solid and liquid } \\ 5 & \text { form. For the first time we demonstrate the low-temperature } \\ 6 & \text { nanosecond pump-probe XANES experiment revealing the } \\ 7 & \text { valence tautomeric transition in a solution of Co complex. } \\ 8 & \text { Reversible transition was induced both under the } \\ 9 & \text { temperature and laser radiation stimuli. }\end{array}$

10 Keywords: valence tautomerism; Co complex; pump11

\section{probe XANES;}

Nowadays, special attention is paid to the development of future data recording, storage and switching molecular devices. ${ }^{1,2,3}$ For these needs, valence tautomeric (VT) metal complexes are actively developed and investigated. ${ }^{4}$ VT compounds undergo conversion-between tautomers due to a reversible intramolecular electron transfer between redox-active ligands and a redox-active metal. Such transitions lead to significant and reversible changes in the structural, optical, and magnetic properties of the metal complexes. Thereby, VT complexes are potential candidates for magneto-optical sensors, switches and molecule-based memory units. ${ }^{5,6}$ Among various VT compounds ${ }^{7,8,9}$ Co complexes with various redox-active quinone ligands have been extensively studied ${ }^{10,11,12}$.

Decades ago D.M. Adams and D.N. Hendrickson have recorded electronic absorption spectra at variable temperatures in toluene of four cobalt bis-o-quinonato complexes of general composition $\left[\mathrm{Co}(3,5-\mathrm{DTBSQ})_{2}\left(\mathrm{~N}^{\wedge} \mathrm{N}\right)\right.$ (where 3,5-DTBSQ- charged forms of 3,5-di-tert-butyl-oquinone and diiminium ligand $\mathrm{N}^{\wedge} \mathrm{N}=1,10$-phenanthroline, 2,2'-dipyridine, 4,4'-dimethyl-dipyridine and 4,4'-diphenyldipyridine) to elucidate the factors controlling the VT interconversion process. ${ }^{13}$ The temperature dependence of the electronic absorption spectra showed dramatic changes with the appearance of isosbestic points during heating and cooling processes. The authors carried out a theoretical analysis of temperature depending VT process in solution using quantum mechanical theory of radiationless transitions. They concluded that the VT transformation is
41 driven by entropy and back transition involves quantum 42 mechanical tunnelling.

As X-ray absorption near-edge structure (XANES) and X-ray emission spectroscopy (XES) are sensitive to the charge state of $3 \mathrm{~d}$ metal and structural rearrangements of organic ligands, they are well-suited methods for VT metal complexes investigation. In the work ${ }^{14}$ authors combined $\mathrm{K}_{\beta}$ XES with Co L-edge XAS to investigate VT states of $\mathrm{Co}$ (phen)-(3,5-DBQ) $)_{2}$ and $\mathrm{Co}$ (tmeda)(3,5-DBQ) complexes. They demonstrated that the low-temperature valence tautomer could be described as a low spin $\mathrm{Co}^{\mathrm{III}}$ and the high-temperature valence tautomer as a high spin $\mathrm{Co}^{\mathrm{II}}$. Herewith, Co $\mathrm{K}_{\beta}$ XES spectra at $375 \mathrm{~K}$ for both complexes showed similar $\mathrm{HS} \mathrm{Co}{ }^{\mathrm{II}}$ electronic configuration. Moreover, the authors observed the occurrence of soft X-ray-induced VT in low-temperature L-edge XAS.

Time-resolved pump-probe XANES is a relatively new and challenging method, which can unravel the nature of short-lived excited states. ${ }^{15,16,17}$ Employing it to the transition metal complexes such as VT materials gives valuable information on excited molecular states and electron transfer processes. ${ }^{18,19,20}$ In recent work ${ }^{21}$ Ryan Ash et al. demonstrated application of $\mathrm{M}_{2,3}$-edge XANES spectroscopy with $40 \mathrm{fs}$ time resolution to study the mechanism and time scale of ultrafast VT in crystalline $\mathrm{Co}^{\mathrm{III}}(\mathrm{Cat}-\mathrm{N}-\mathrm{SQ})(\mathrm{Cat}-\mathrm{N}-\mathrm{BQ})$ complex. Their result showed that ultrafast core-level M-edge XANES allows separation of vibrational and electronic relaxation transition from hot $\mathrm{HS} \mathrm{Co}^{\mathrm{II}}$ state to the LS Co ${ }^{\mathrm{III}}$ ground state. However, there is still just a few works that provided transient XANES data for Co systems with VT.

Moreover, to the best of our knowledge, a pump-probe XANES experiment with VT complexes dissolved in liquid has not yet been demonstrated. Both liquid and solid state experiments are essential since the effects of intermolecular interactions affect the parameters of the VT transition, such as temperature, hysteresis and intermediate phases. ${ }^{22}$ In our work, we present the technical feasibility of such an experiment for the Co complex with (N-cyclo-hexyl-2iminopyridine)(3,6-di-tert-butyl-o-

benzosemiquinonato)(3,6-di-tert-butyl-catecholato) ligand. 
1 Laser pump - X-ray probe study was performed at the 2 Super-XAS beamline of the Swiss Light Source, Villigen, 3 PSI. We used the Si(111) monochromator and asynchronous 4 X-ray photon counting regime, ${ }^{23}$ which provided $20 \mathrm{~ns}$ time 5 resolution. A tungsten foil with a $50 \mu \mathrm{m}$ pinhole was placed 6 on the nozzle to allow laser/X-ray alignment. We placed 7 Avalanche photodiode (APD) fluorescent - detector with a 8 Z-1 filter and Soller slits close to the jet. A green 9 nanosecond laser (DS20HE-532 from Photonics Industries) 10 with $532 \mathrm{~nm}$ wavelength was operated at a $150 \mathrm{kHz}$ 11 repetition rate. Full laser power before the sample chamber 12 was measured as $6 \mathrm{~W}$. Laser pulse width was 20 ns. Co K13 edge spectra were initially measured in a static mode before 14 and after cooling to confirm the VT transition. The X-ray 15 photon counting scheme was changed then to time-tagged 16 photon counting, and pump-probe spectra were registered in 17 the asynchronous mode under pulsed laser illumination at 18 the lowest temperature

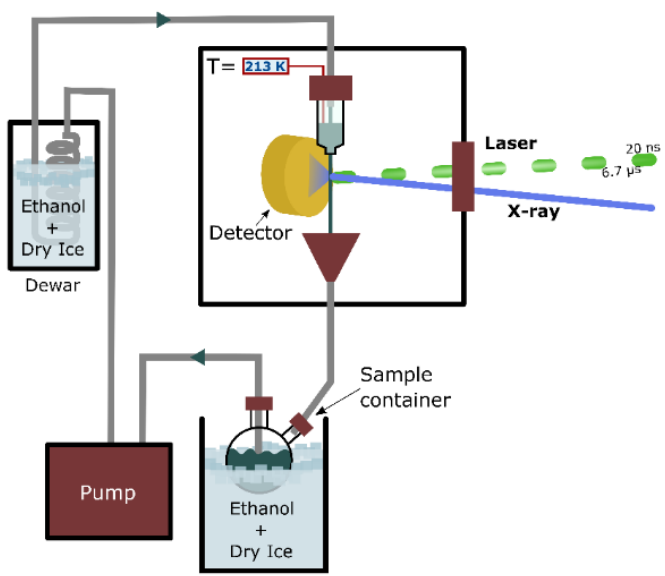

Figure 1. Scheme of experimental pump-probe XAS setup at the Super-XAS beamline, Swiss Light Source.

The scheme of the experiment is presented in Figure 1. The cooling system consisted of two Dewar vessels filled with ethanol and dry ice. The first Dewar contained a vessel with the sample solution in toluene (the total volume of the sample circulating in the system was about $80 \mathrm{ml}$ ). The second Dewar contained a coil pipe with the circulating sample. The opened part (nozzle) of the jet was inside the gas-tight chamber.

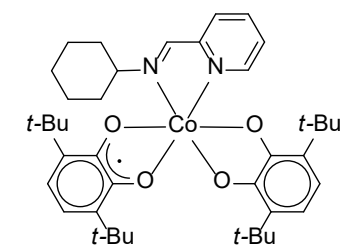

Complex Co2 (Figure 2) was synthesized by exchange reaction of tris- $O$-semiquinonato cobalt complex with corresponding iminopyridine (Figure S1).

$\mathrm{We}$ carried on a low-temperature UV-Vis spectroscopic study of $\mathbf{C o 2}$ complex dissolved in toluene in transmittance mode using a custom assembled cell. Gradual changes in optical properties were occurring upon decreasing temperature (Figure 3). The optical absorption spectra indicated a VT transition similar to that demonstrated in. ${ }^{13,24}$

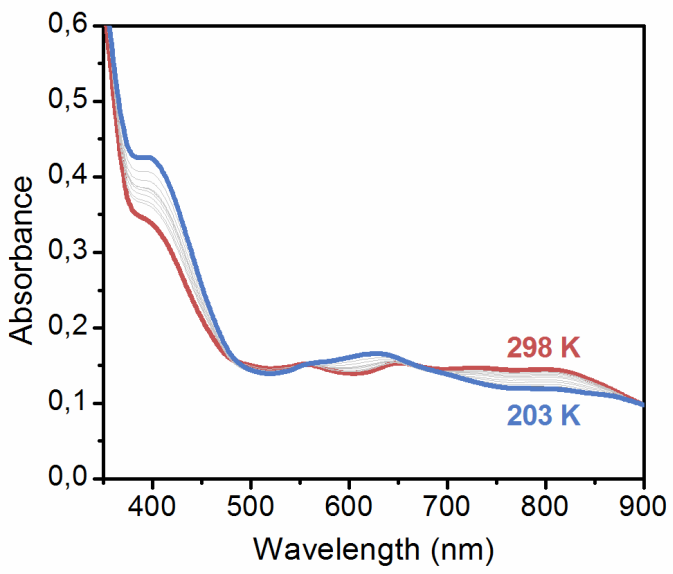

Figure 3. Temperature resolved UV-Vis spectra of the Co2 toluene solution.

At lower temperatures, the intensity of a broad band at $800 \mathrm{~nm}$ decreases, while bands at near $600 \mathrm{~nm}$ and $395 \mathrm{~nm}$ increase. The band at $700-850 \mathrm{~nm}$ is characteristic of the $\mathrm{HS} \mathrm{Co}^{\mathrm{II}}$ tautomer and has likely a metal-to-ligand charge transfer nature. ${ }^{13,24}$ At $203 \mathrm{~K}$ the peak at $\sim 620 \mathrm{~nm}$ characterises the $\mathrm{LS} \mathrm{Co}{ }^{\mathrm{III}}$ tautomer. It originates from the ligand-to-metal charge transfer. ${ }^{25}$ The band at $395 \mathrm{~nm}$ increases along with sample cooling and includes the $\pi-\pi^{*}$ ligand-based transition in the LS $\mathrm{Co}^{\mathrm{III}}$ tautomer. ${ }^{26,27}$ The appearance of isosbestic points at near $560 \mathrm{~nm}$ and $657 \mathrm{~nm}$ during cooling is strong evidence that only two different states were present in the solution. ${ }^{13}$

VT transition in solution is observed at lower temperatures compared to solid state (see Figure 4 below). The magnetic moment of $\mathbf{C o 2}$ in crystal slowly increases from $1.7 \mu_{\mathrm{B}}$ at $50 \mathrm{~K}$ to $2.1 \mu_{\mathrm{B}}$ at $260 \mathrm{~K}$ (Figure $4 \mathrm{a}$ ). It decreases to $0.75 \mu_{\mathrm{B}}$ upon cooling to $2.0 \mathrm{~K}$ due to intermolecular antiferromagnetic interactions. The beginning of VT transformation is observed as fast increase of the magnetic momentum above $260 \mathrm{~K}$.

FTIR spectra in the Figure $4 \mathrm{~b}$ further confirm the VT transition in the crystalline phase above room temperature. In contrast to the UV-vis data measured in solution, no evident changes occur in FTIR spectra upon cooling. In agreement with magnetic moment measurements, the crystalline sample at room temperature is characterized as the LS Co ${ }^{\mathrm{III}}$ state. Spectra recorded during heating above 
a
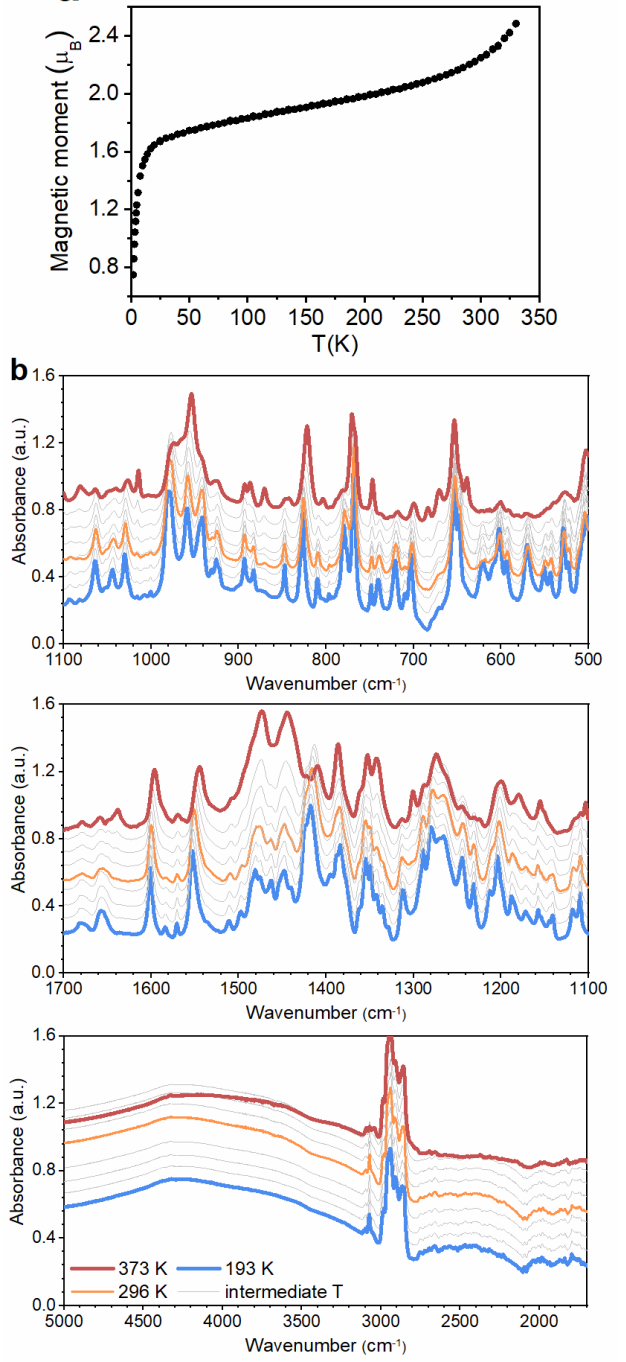

Figure 4. (a) Magnetic moment (in solid) and (b) temperatureresolved FTIR spectra for the powder in the range $193 \mathrm{~K}-373 \mathrm{~K}$.

$363 \mathrm{~K}$ show noticeable changes in the peak intensities and positions. Two peaks at $1600 \mathrm{~cm}^{-1}$ and $1550 \mathrm{~cm}^{-1}$, associated with the vibrations of the pyridine cycle, shift towards lower wavenumbers. ${ }^{22}$ Features in the $1500-1400 \mathrm{~cm}^{-1}$ region characterize bond stretch vibrations of one-and-a-half $\mathrm{C}-\mathrm{O}$ bonds in semiquinone (SQ) ligand ${ }^{22}$ and reveal strong changes at elevated temperatures, thus confirming ligand-tometal electron transfer coupled with the VT transformation. Three intense peaks at $900-1000 \mathrm{~cm}^{-1}$ merge into the band with one sharp peak at $953 \mathrm{~cm}^{-1}$. The broad band in the near-infrared region $\left(5000-3500 \mathrm{~cm}^{-1}\right)$ increases in intensity with cooling and decreases upon heating. It is associated with the $\mathrm{LS} \mathrm{Co}^{\mathrm{III}}$ tautomer and originates from the mixed-valence intervalence charge transfer involving excitation of an electron from the $\mathrm{Cat}^{2-}$ to the $\mathrm{SQ}^{-}$ligand. ${ }^{13,28}$

Quantum chemical calculations were performed to describe structural changes in the complex associated with the VT transition. The modelling was performed within density functional theory (DFT) in Gaussian 16. ${ }^{29}$ Three functionals were compared, namely PBE0, ${ }^{30} \mathrm{~B} 3 \mathrm{LYP} * 31-33$ and TPSSh ${ }^{34,35}$ using Def2-TZVP basis set. ${ }^{36}$ The calculated spin density distribution $\left(\mathrm{q}_{\mathrm{s}}^{\mathrm{Co}}\right)$ is presented in Table $\mathrm{S} 1$. High-spin structures contain three unpaired electrons on the cobalt ion, which indicates the $\mathrm{HS} \mathrm{Co}^{\mathrm{II}}$ state of the metal. For the low-spin state of the molecule, this parameter does not exceed 0.1 , thus confirming the $\mathrm{LS} \mathrm{Co}^{\mathrm{III}}$ state of the metal. The relative energies of the isomers calculated with the PBE0 and B3LYP* functionals show the energetic preference of the high-spin state ( $\mathrm{HS} \mathrm{Co}^{\mathrm{II}}$ ), which is inconsistent with the existence of VT in the complex. Correct results were obtained with the TPSSh functional only. The values of the operator $\left\langle\mathrm{S}^{2}>\right.$ given in the Table S1 deviate slightly from those expected for the corresponding spin states (8.75 and 0.75), which indicates slight spin contamination (mixing of spin states) in the obtained structures.

In contrast to the controversial values of the HS-LS energy differences the DFT calculations predict similar values of the bond lengths between the metal-donor atoms for all three functionals (Table S2). The variation of these distances between the two spin states is in agreement with the expected VT transition. Smaller Co-O,N bond lengths in the $\mathrm{LS} \mathrm{Co}{ }^{\mathrm{III}}$ state are observed for all applied functionals. For all geometries the bond length $\mathrm{Co}-\mathrm{N}$ is always longer than Co-O. Both observations are the consequence of Coulomb interactions of $\mathrm{Co}^{3+}-\mathrm{Cat}^{2-}$ versus $\mathrm{Co}^{2+}-\mathrm{SQ}^{-}$ where slightly negatively charged iminopyridine affects the Co-N bonds lengths.

We measured cobalt K-edge XANES during cooling of the sample dissolved in toluene. In agreement with UVvis measurements, the cobalt oxidation and spin state change below $240 \mathrm{~K}$ (Figure 5a). The shift of all spectral features to the higher energies upon cooling indicates valence state transition accompanied by strong structural changes in the complex. ${ }^{37}$ According to the semi-empirical Natoli's rule, this shift indicates that the transformation from $\mathrm{HS} \mathrm{Co}^{\mathrm{II}}$ to $\mathrm{LS} \mathrm{Co} \mathrm{Co}^{\mathrm{III}}$ induces the shortening of $\mathrm{Co}-\mathrm{O}$ and Co-N bonds, in agreement with the DFT calculations and EXAFS analysis (Table S3).

The Co K-edge XANES spectra were calculated for the optimized structures using finite difference approach and compared to the experimental data (Figure S2). All theoretical spectra independently on the chosen functional show a good agreement with experimental data. The most significant deviation was observed for the LS Co ${ }^{\mathrm{III}}$ geometry obtained within B3LYP* approximation. As the best result we have chosen the spectra calculated for TPSSh functional (Figure 5a) since only this potential provides correct energy values.

The next question that we have addressed was about the Co charge and spin state in the LS Co ${ }^{\mathrm{III}}$ complex after photoexcitation. Pump-probe XAS has a high sensitivity and possibility to probe short-lived states with a lifetime in different time domains. Since the initial LS $\mathrm{Co}^{\mathrm{III}}$ state is accumulated at low temperature, we have modified the setup to enable experiments for liquid samples at low temperature. 
1 Excitation has been performed at $532 \mathrm{~nm}$, which is the high 2 energy side of the LMCT band. Data obtained using the 3 pump-probe method are shown in Figure 5b.
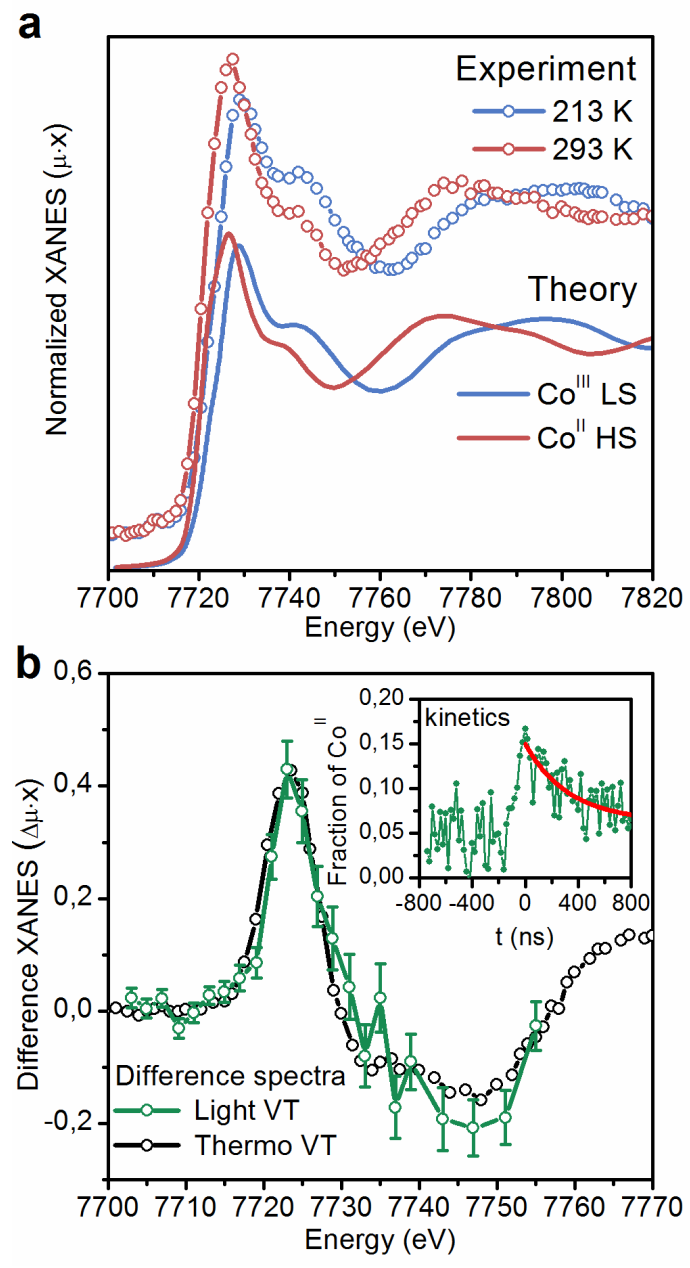

Figure 5. (a) Co K-edge XANES spectra measured for Co2 sample at $213 \mathrm{~K}$ and $293 \mathrm{~K}$ in a toluene solution and calculated for DFT-optimized structures within TPSSh functional (b) averaged transient difference XANES signal after laser excitation for Co2. The shape of the transient did not vary in the studied time delay range. Inset: kinetics of the amplitude of difference signal.

Principal component analysis of the spectra series measured with $20 \mathrm{~ns}$ step has indicated that only two components were present: initial LS Co ${ }^{\mathrm{III}}$ state and the photo-induced state. From the comparison of the pumpprobe transient spectrum with the difference between HS $\mathrm{Co}^{\mathrm{II}}$ and $\mathrm{LS} \mathrm{Co}^{\mathrm{III}}$ states obtained by changing the temperature, we can conclude that HS state was formed due to photoexcitation. Within our time resolution, we do not see any signatures of LMCT state before spin transition (LS $\mathrm{Co}^{\mathrm{II}}$ ), which should have a significantly different local structure compared to $\mathrm{HS} \mathrm{Co}^{\mathrm{II}}$ and therefore easily distinguishable XAS spectrum. The lifetime of photoinduced $\mathrm{HS} \mathrm{Co}^{\mathrm{II}}$ state estimated through exponential fitting as $250 \pm 50 \mathrm{~ns}$ (Figure $5 \mathrm{~b}$ inset, red line is a monoexponential decay fit) agrees with values reported for related compounds obtained using the optical pump-probe method. ${ }^{13}$

In conclusion, we observed valence tautomeric transition for Co2 complex with N-cyclohexyl-2iminopyridine and 3,6-di-tert-butyl-o-benzosemiquinonato ligands upon temperature variation both in a liquid form and a powder. In agreement with magnetic moment measurements, FTIR spectra demonstrated noticeable changes only above $363 \mathrm{~K}$. The solid sample at room temperature was characterized as the $\mathrm{LS} \mathrm{Co}^{\mathrm{III}}$ statewhile the optical absorption spectra and Co K-edge XANES spectra in the toluene solution confirmed the $\mathrm{HS} \mathrm{Co}^{\mathrm{II}}$ state. At $213 \mathrm{~K}$ we registered a transient laser pump - X-ray probe signal in the $\mathbf{C o 2}$ toluene solution that reproduced the difference between temperature stabilized HS and LS states. The relaxation kinetics to the ground state occured with a time constant of $250 \pm 50 \mathrm{~ns}$. Our results indicate that reversible transition in the studied complex could be induced both under the influence of temperature and laser radiation. This experiment opens new perspectives for the pump-probe experiments for liquid samples at low temperatures.

Pump-probe measurements were carried out within the framework of the project of the Russian Foundation for Basic Research (RFBR \#18-02-40029). The synthetic work was performed in the framework of the Russian state assignment. The authors are thankful to A.S. Bogomyakov for magnetic measurements.

Supporting Information is available on http://dx.doi.org/10.1246/cl.******.

\section{References and Notes}

P. Gütlich, Y. Garcia, T. Woike, Coord. Chem. Rev. 2001, 219221,839

A. P. de Silva, N. D. McClenaghan, Chemistry - A European Journal 2004, 10 (3), 574.

3 K. V. Raman, A. M. Kamerbeek, A. Mukherjee, N. Atodiresei,T. K. Sen, P. Lazić, V. Caciuc, R. Michel, D. Stalke, S. K. Mandal, et al. Nature 2013, 493 (7433), 509.

4 T. Tezgerevska, K. G. Alley, C. Boskovic, Coord. Chem. Rev. 2014, 268, 23.

5 A. Calzolari, Y. Chen, G. F. Lewis, D. B. Dougherty, D. Shultz, M. Buongiorno Nardelli, The Journal of Physical Chemistry B 2012, 116 (43), 13141.

6 O. Sato, J. Tao, Y.-Z. Zhang, Angewandte Chemie International Edition 2007, 46 (13), 2152.

7 J. Bendix, K. M. Clark, Angewandte Chemie International Edition 2016, 55 (8), 2748.

8 H. W. Liu, K. Matsuda, Z. Z. Gu, K. Takahashi, A. L. Cui, R. Nakajima, A. Fujishima, O. Sato, Physical Review Letters 2003, 90 (16), 167403.

9 M. J. Sever, J. J. Wilker, Dalton Transactions 2004, 1061.

10 A. Caneschi, A. Cornia, A. Dei, Inorg. Chem. 1998, 37 (13), 3419.

11 O.-S. Jung, D. H. Jo, Y.-A. Lee, Conklin, B. J.; Pierpont, C. G. Inorg. Chem. 1997, 36 (1), 19.

12 D. Ruiz-Molina, J. Veciana, K. Wurst, D. N. Hendrickson, C. Rovira, Inorg. Chem. 2000, 39 (3), 617.

13 D. M. Adams, D. N. Hendrickson, J. AM. CHEM. SOC. 1996, $118(46), 11515$. 
14 H. W. Liang, T. Kroll, D. Nordlund, T.-C. Weng, D. Sokaras, C. G. Pierpont, K. J. Gaffney, Inorg. Chem. 2017, 56 (2), 737.

15 M. L. Shelby, P. J. Lestrange, N. E. Jackson, K. Haldrup, M. W. Mara, A. B. Stickrath, D. Zhu, H. Lemke, M. Chollet, B. M. Hoffman, X. Li, L. X. Chen, J. Am. Chem. Soc. 2016, 138, 28, 8752 .

16 G. Smolentsev, B. Cecconi, A. Guda, M. Chavarot-Kerlidou, J. A. van Bokhoven, M. Nachtegaal, V. Artero, Chem. Eur. J. 2015, 21, 15158 .

17 A. Cannizzo, C.J. Milne, C. Consani, W. Gawelda, Ch. Bressler, F. van Mourik, M. Chergui, Coordination Chemistry Reviews, 2010, 254, 2677.

18 C. Bressler, C. Milne, V. T. Pham, A. ElNahhas, R. M. van der Veen, W. Gawelda, S. Johnson, P. Beaud, D. Grolimund, M. Kaiser, et al. Science 2009, 323 (5913), 489.

19 S. Della-Longa, L. X. Chen, P. Frank, K. Hayakawa, K. Hatada, M. Benfatto, Inorg. Chem. 2009, 48 (9), 3934.

20 K. Hong, H. Cho, R. W. Schoenlein, T. K. Kim, N. Huse, Accounts of Chemical Research 2015, 48 (11), 2957.

21 R. Ash, K. Zhang, J. Vura-Weis, The Journal of Chemical Physics 2019, 151 (10), 104201.

22 A. A. Guda, M. Chegerev, A. G. Starikov, V. G. Vlasenko, A. A. Zolotukhin, M. P. Bubnov, V. K. Cherkasov, V. V. Shapovalov, Yu. V. Rusalev, A. A. Tereshchenko, A. L. Trigub, A. V. Chernyshev, A. V. Soldatov, J. Phys.: Condens. Matter, 2021,33, 215405.

23 G. Smolentsev, A. A. Guda, M. Janousch, C. Frieh, G. Jud, F. Zamponi, M. Chavarot-Kerlidou, V. Artero, J. A. van Bokhoven, M. Nachtegaal, Faraday Discussions 2014, 171 (0), 259.

24 D. M. Adams, A. Dei, D. N. Hendrickson, A. L. Rheingold, A. Dei, J. AM. CHEM. SOC. 1993, 115 (18), 8221.

25 D. M. Adams, B. Li,; J. D. Simon, D. N. Hendrickson, Angew. Chem. Int. Ed. Engl. 1995, 34 (13-14), 1481.

26 G. K. Gransbury, M.-E. Boulon, S. Petrie, R. W. Gable, R. J. Mulder, L. Sorace, R. Stranger, C. Boskovic, Inorg. Chem. 2019, $58(7), 4230$.

27 T. Mibu, Y. Suenaga, T. Okubo, M. Maekawa, T. Kuroda-Sowa, Inorganic Chemistry Communications 2020, 114, 107826.

28 Cui, A.; Takahashi, K.; Fujishima, A.; Sato, O. J. Photochem. Photobiol. A Chem. 2004, 167 (2), 69.

29 M. J. Frisch, G. W. Trucks, H. B. Schlegel, G. E. Scuseria, M. A. Robb, J. R. Cheeseman, G. Scalmani, V. Barone, G. A. Petersson, H. Nakatsuji, et al. Wallingford, CT, 2016.

30 J. P. Perdew, K. Burke, M. Ernzerhof, Physical Review Letters 1996, 77 (18), 3865.

31 A. D. Becke, Physical Review A 1988, 38 (6), 3098.

32 A. D. Becke, The Journal of Chemical Physics 1993, 98 (2), 1372 .

33 C. Lee, W. Yang, R. G. Parr, Physical Review B 1988, 37 (2), 785 .

34 J. P. Perdew, Tao J Fau - V. N. Staroverov, Staroverov Vn Fau G. E. Scuseria, G. E. Scuseria, (0021-9606 (Print)).

35 J. P. Perdew, S. Kurth, A. Zupan, P. Blaha, Physical Review Letters 1999, 82 (12), 2544.

36 F. Jensen, WIREs Computational Molecular Science 2013, 3 (3), 273.

37 P. Glatzel, G. Smolentsev, G. Bunker, J. Phys.: Conf. Ser. 2009, $190,012046$. 
The diagram is acceptable in a colored form. Publication of the colored G. $A$. is free of charge.

For publication, electronic data of the colored G.A. should be submitted. Preferred data format is EPS, PS, CDX, PPT, and TIFF.

If the data of your G.A. is "bit-mapped image" data (not "vector data"), note that its print-resolution should be 300 dpi.

You are requested to put a brief abstract (50-60words, one paragraph style) with the graphical abstract you provided, so that readers can easily understand what the graphic shows.

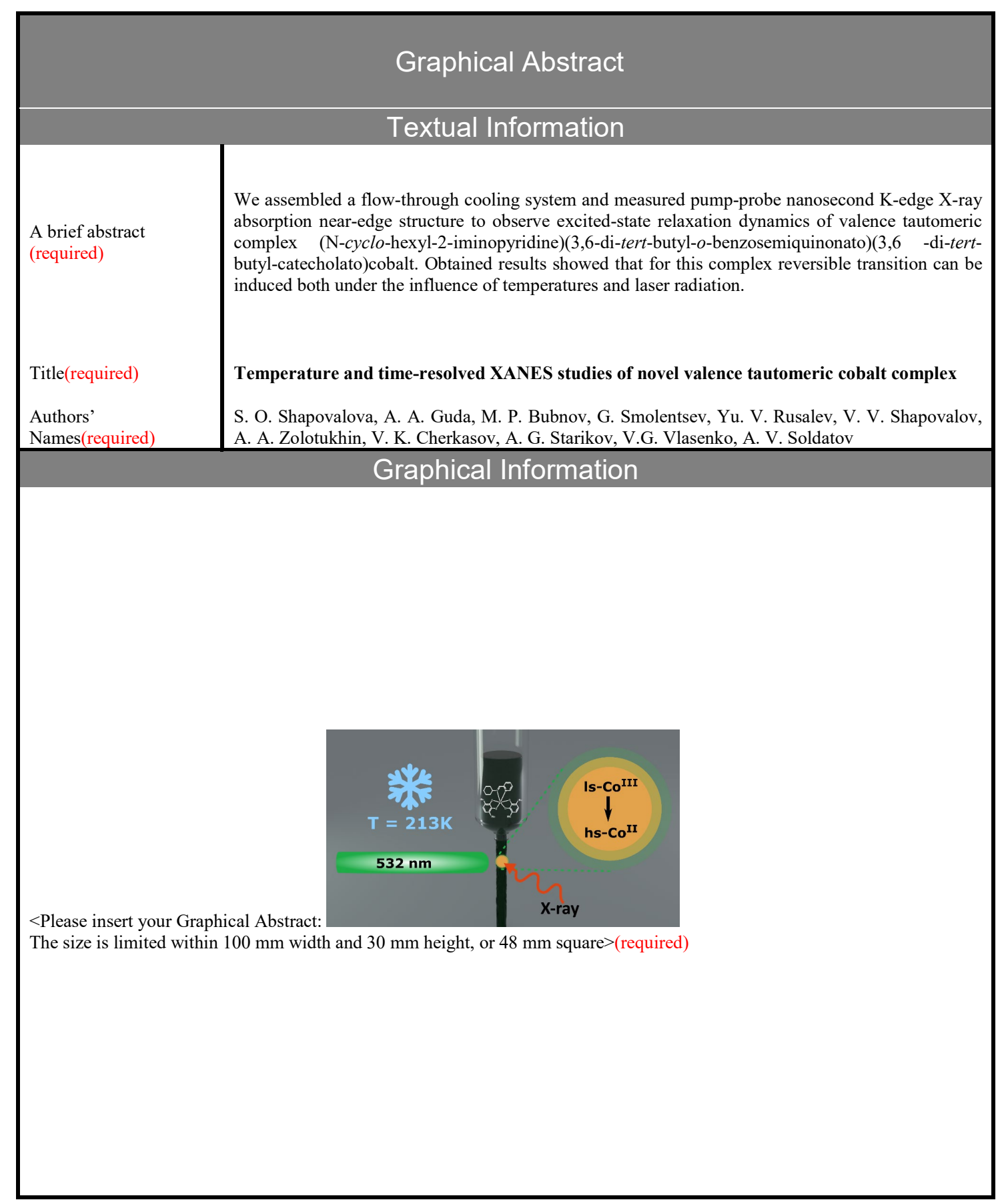

